\title{
Papers
}

\section{Use of automated external defibrillator by first responders in out of hospital cardiac arrest: prospective controlled trial}

Anouk P van Alem, Rob H Vrenken, Rien de Vos, Jan G P Tijssen, Rudolph W Koster

\begin{abstract}
Objective To test the hypothesis that the use of an automated external defibrillator by police and fire fighters results in higher discharge rates for out of hospital cardiac arrest.

Design Controlled clinical trial with initial random allocation of automated external defibrillators to first responders in four of the eight participating regions; each region switched from control to experimental, and vice versa, every four months. Setting Amsterdam and surroundings, the Netherlands. Participants Patients with witnessed out of hospital cardiac arrests, identified by the emergency medical system between January 2000 and January 2002.

Main outcomes measures Survival to hospital discharge; return of spontaneous circulation; admission to hospital. Results 243 patients (65\% in ventricular fibrillation) were included in the experimental area and 226 patients $(67 \%$ in ventricular fibrillation) in the control area. The median time interval between collapse and first shock was 668 seconds in the experimental area and 769 seconds in the control area $(\mathrm{P}<0.001) .44(18 \%)$ patients in the experimental area versus $33(15 \%)$ patients in the control area were discharged (odds ratio $1.3(95 \%$ confidence interval 0.8 to 2.2$), \mathrm{P}=0.33), 139$ (57\%) experimental versus $108(48 \%)$ control patients had return of spontaneous circulation (1.5 (1.0 to 2.2), $\mathrm{P}=0.05$ ), and $103(42 \%)$ experimental versus $74(33 \%)$ control patients were admitted (1.5 (1.1 to 1.6), $\mathrm{P}=0.02$ ). The median delay from receipt of call to dispatch of the ambulance was 120 seconds, and the delay to dispatch of the first responder was 180 seconds.

Conclusions Use of automated external defibrillators by first responders did not significantly increase survival to discharge from hospital, although it did improve return of spontaneous circulation and admission to hospital. Improved dispatch procedures should increase the success of programmes of first responders using external defibrillators.
\end{abstract}

\section{Introduction}

Automated external defibrillators (AEDs) are computerised devices that are reliable and simple to operate, enabling trained lay rescuers to perform defibrillation as a key element in resuscitation effort. ${ }^{1-4}$ Initial uncontrolled reports showed that police officers used the AED skilfully and successfully. ${ }^{56}$ Two recent studies of dispatched police suggested improved survival from out of hospital cardiac arrest. ${ }^{78}$ The 2000 guidelines for cardiopulmonary resuscitation and emergency cardiovascular care consider use of the AED to be a class IIa recommendation for level one responders such as police and fire fighters if the emer- gency medical system cannot achieve a first shock within five minutes of the call. ${ }^{4}$ A class IIa recommendation indicates that the weight of evidence or opinion is in favour of usefulness, efficacy, or both. No prospective randomised controlled clinical trial has been done to test the benefit of the use of AEDs by dispatched first responders.

We did a controlled clinical trial with systematic allocation of AEDs. This allowed us to test the hypothesis that introduction of AEDs used by dispatched first responders in a standard emergency medical system will result in higher discharge rates for out of hospital cardiac arrest.

\section{Methods}

Study design

We designed the study as an open clinical trial, with controlled allocation of AEDs. We included patients with witnessed cardiac arrest in whom resuscitation was attempted. We excluded patients below the age of 18 years, patients with a cardiac arrest of traumatic origin, and patients whose cardiac arrest was witnessed by the emergency medical system. The study area included the city of Amsterdam and a surrounding area with urban and rural parts, including 1.6 million inhabitants and covering $885 \mathrm{~km}^{2}$.

\section{Randomisation}

We achieved randomisation by systematic allocation of AEDs. We divided the study area into two regions with the fire brigade as first responders and six districts with police as first responders. The experimental area consisted of one fire brigade region and three police districts, equipped with 50 AEDs (LIFEPAK 500 AED, Medtronic Physio-Control, Redmond, WA, USA). The other fire brigade region and police districts served as the control area. Every four months we collected all the AEDs from the experimental area and distributed them in the control area, which then served as the experimental area for the next four months. With this procedure we tried to ensure an even distribution of external characteristics such as population, socioeconomic status, hospitals, response times, and seasons between the two groups. In total, each region served 12 months as the experimental area and 12 months as the control area.

\section{Emergency medical system}

When the emergency medical system dispatch centre suspected a cardiac arrest, it dispatched two ambulances and then immediately alerted the police or fire brigade dispatch centre. All ambulances were manned by a nurse or paramedic and a driver, equipped with a manual defibrillator and qualified to perform advanced cardiopulmonary life support. After receiving the call 
from the dispatch centre, the police or fire dispatch centre directed a police patrol car or fire engine to the scene. Immediately thereafter one of our dedicated data collectors (a team of trained fifth year medical students working in shifts covering 24 hours a day) travelled to the scene independently from the emergency medical system or first responders. Neither the firemen nor the police officers had ever responded to medical emergencies before, but they were trained in cardiopulmonary resuscitation.

All fire and police districts were adjacent without geographical overlap. Police were dispatched in both experimental and control periods, and the fire brigade was dispatched during experimental periods only.

When first responders reached the scene of the arrest first, in an area serving as experimental area, they would perform cardiopulmonary resuscitation and use the AED. When the ambulance personnel arrived at the scene they would take over the resuscitation and use their manual device. When the ambulance personnel arrived first at the scene, they started the resuscitation and first responders assisted in cardiopulmonary resuscitation if necessary. The protocol for delivery of shocks was $200 \mathrm{~J}$, followed by $200 \mathrm{~J}$, and then $360 \mathrm{~J}$ for each shock thereafter as needed for defibrillation.

\section{Training first responders}

For the study, 1063 police officers and 586 fire fighters were trained in the use of the AED, and their cardiopulmonary resuscitation skills were refreshed. Officers were trained in pairs in 3.5 hour sessions. Refresher training was conducted at eight months intervals.

\section{Data collection}

Data collectors collected the data at the scene. They collected data on the circumstances of the cardiac arrest, the estimated moment of the collapse, witnesses, cardiopulmonary resuscitation by bystanders, sequence of events, and relevant time points by using a standardised method to directly interview all people involved. They downloaded the electrocardiogram and voice recordings from the AED and the electrocardiogram of the manual defibrillator. They corrected deviations of internal clocks by comparison with radio controlled wristwatches. Time of call, dispatching, and arrival on scene of first responders and ambu- lance were obtained from time corrected dispatch computers. Date of death or discharge was obtained from hospital records.

\section{Endpoints}

The primary endpoint of the study was survival to hospital discharge. Secondary endpoints were return of spontaneous circulation and admission to hospital. We analysed all endpoints on an intention to treat basis, irrespective of whether first responders or ambulance personnel performed defibrillation.

\section{Power calculation}

The study was powered to show an absolute difference in survival between the two groups of $12 \%$ (from $8 \%$ to $20 \%$ ), assuming a decrease in the median time interval from collapse to defibrillation from 11 minutes to six minutes. We assumed that ventricular fibrillation would be the initial rhythm in $60 \%$ of patients and that the probability of survival was 33\% when the first shock was given in six minutes after collapse or 13\% when the first shock was given after 11 minutes. Survival rate in any other rhythm was expected to be $1.8 \%$. We expected cardiopulmonary resuscitation by a bystander to occur in $30-50 \%$ of patients. We based all assumptions on previous observations. ${ }^{9}$ With $\alpha=0.05$ and $\beta=0.05$, we needed 210 patients per group.

\section{Statistical methods}

Time intervals are expressed as medians and interquartile ranges. We tested significance with $\chi^{2}$ statistics for proportions and the Mann-Whitney $\mathrm{U}$ test for continuous variables. We accepted significance when a two sided $\mathrm{P}$ value was $<0.05$ or confidence intervals did not include unity. We used logistic regression models to analyse the three binary endpoints. In order to match the analyses to the design of the study, we extended the logistic regression models with the generalised estimating equations method. ${ }^{10} 11$ This approach uses weighted combinations of observations to extrapolate the appropriate amount of information from correlated data, ${ }^{12}$ and allowed us to take into account any correlation that might arise from factors related to region or period. We used the generalised linear model procedure (proc genmod) in SAS version 8.2 to do this. We used SPSS 10.0 for Apple Macintosh for all other analyses.

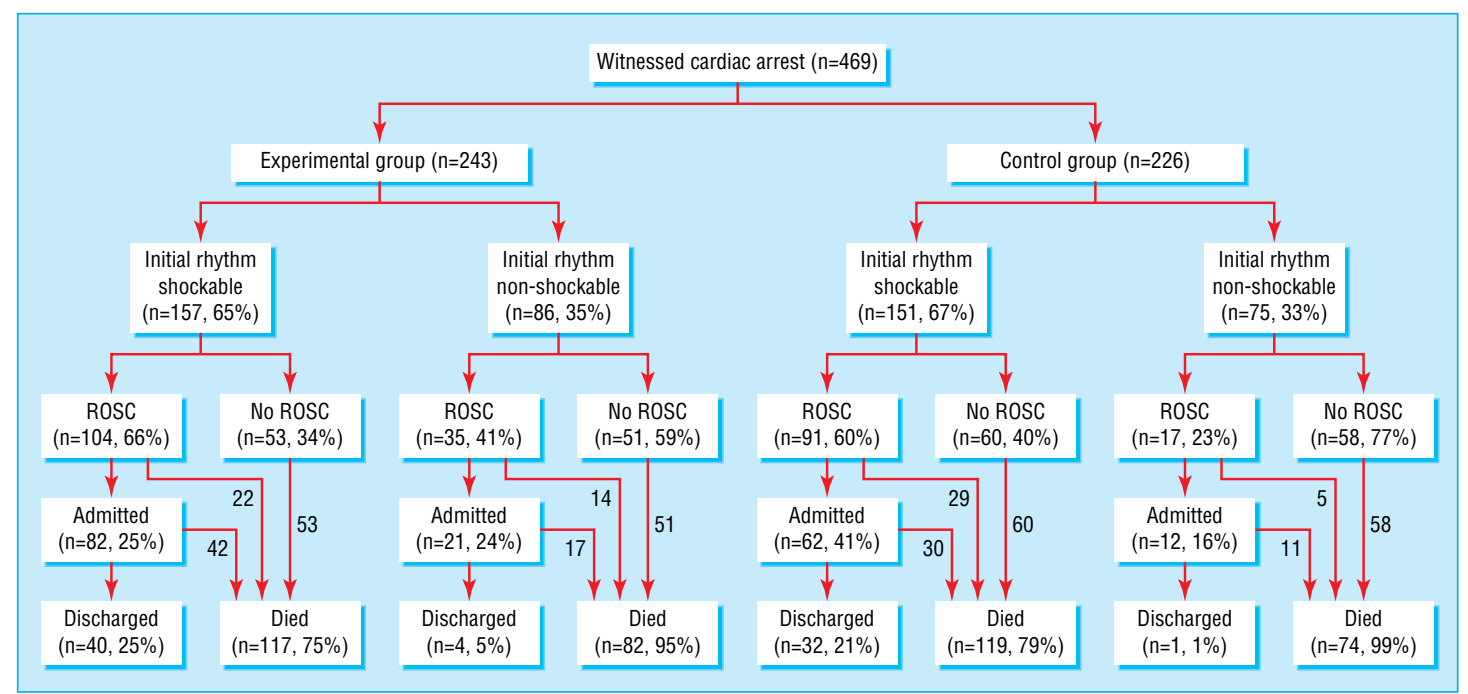

Fig 1 Primary and secondary outcomes according to treatment group, stratified according to initial rhythm. Admission denotes admission to hospital after leaving the emergency room; ROSC=return of spontaneous circulation 
Table 1 Characteristics of included patients with out of hospital cardiac arrest. Values are numbers (percentages) unless stated otherwise

\begin{tabular}{|c|c|c|}
\hline Characteristic & Experimental group $(\mathrm{n}=243)$ & Control group ( $\mathrm{n}=226)$ \\
\hline Mean (SD, range) age in years & $67(14,29-93)$ & $65(14,21-90)$ \\
\hline \multicolumn{3}{|l|}{ Sex: } \\
\hline Female & $56(23)$ & $54(24)$ \\
\hline Male & $187(77)$ & $172(76)$ \\
\hline Mean (SD, range) weight in $\mathrm{kg}$ & $80(16,55-130)$ & $82(17,55-135)$ \\
\hline \multicolumn{3}{|l|}{ Witnessed by: } \\
\hline Partner & $129(53)$ & $119(53)$ \\
\hline Bystander or relative & $107(44)$ & $94(42)$ \\
\hline General practitioner & $4(2)$ & $11(5)$ \\
\hline Police & $3(1)$ & $2(1)$ \\
\hline \multicolumn{3}{|l|}{ Location of collapse: } \\
\hline Home & $173(71)$ & $162(72)$ \\
\hline Public place & $37(15)$ & $31(14)$ \\
\hline Public road & $33(14)$ & $31(14)$ \\
\hline Long term care facility & 0 & $2(1)$ \\
\hline Basic CPR performed & $114(47)$ & $119(53)$ \\
\hline By partner & $20(18)$ & $19(16)$ \\
\hline By bystander or relative & $80(70)$ & $70(59)$ \\
\hline By medical professional & $14(12)$ & $30(25)$ \\
\hline First responder CPR before arrival of ambulance & $74(30)$ & $61(27)$ \\
\hline \multicolumn{3}{|l|}{ Median (IQR) time interval in seconds: } \\
\hline Collapse to start of basic $\mathrm{CPR}^{\star} \dagger$ & $90(60-210)$ & $90(60-180)$ \\
\hline Collapse to first shock $\dagger$ & 668 (529-939) & 769 (607-981) \\
\hline Collapse to ACLS† & $840(690-1140)$ & $840(660-1020)$ \\
\hline
\end{tabular}

ACLS=advanced cardiopulmonary life support; CPR=cardiopulmonary resuscitation; IQR=interquartile range.

*Based on patients who received cardiopulmonary resuscitation from a bystander before arrival of ambulance.

†Based on patients with shockable initial rhythm.

Table 2 Primary and secondary outcomes according to treatment group of patients in shockable and non-shockable initial rhythm. Values are numbers (percentages) unless stated otherwise

\begin{tabular}{|c|c|c|c|c|}
\hline Outcome & Experimental group $(\mathrm{n}=243)$ & Control group $(\mathrm{n}=226)$ & Odds ratio* $(95 \% \mathrm{CI})$ & $P$ value \\
\hline Survival to discharge from hospital & $44(18)$ & $33(15)$ & $1.3(0.8$ to 2.2$)$ & 0.33 \\
\hline Return of spontaneous circulation & $139(57)$ & $108(48)$ & 1.5 (1.0 to 2.21$)$ & 0.05 \\
\hline Admission to hospital & $103(42)$ & $74(33)$ & 1.5 (1.1 to 2.0$)$ & 0.02 \\
\hline
\end{tabular}

${ }^{*}$ Derived from the generalised estimating equations model.

\section{Results}

Between 5 January 2000 and 5 January 2002905 patients had a suspected cardiac arrest. In 214 patients cardiac arrest was not present at arrival of help, and in 108 patients no resuscitation was attempted. Of the patients in whom resuscitation was attempted, 114 were not witnessed. A total of 469 patients met the criteria for inclusion: 243 patients in the experimental area and 226 patients in the control area. Table 1 shows the characteristics of these two groups. None of the differences was statistically significant, except the time interval between collapse and first shock $(\mathrm{P}<0.001)$.

Figure 1 and table 2 show the results for the primary and secondary endpoints, comparing experimental and control groups. The observed difference in survival could indicate that in a community where first responders are equipped with AEDs, 33 dispatches for a witnessed arrest could result in the added survival to discharge of one patient (number needed to treat).

For four patients in the experimental group and three patients in the control group, the first responders witnessed or encountered the arrest without being dispatched. In the experimental group, three of these four patients survived to discharge. In the control group none of the three patients survived.

Figure 2 shows the cumulative time intervals for the process of call, dispatch, and arrival of the ambulance and first responders. Figure 3 shows the cumulative time intervals between collapse and first shock for the experimental and control groups.
The median time gain to first shock was 1 minute and 41 seconds in favour of the experimental group $(\mathrm{P}<0.001)$.

In the experimental group, $72(46 \%)$ patients with a shockable rhythm were initially shocked by first responders and

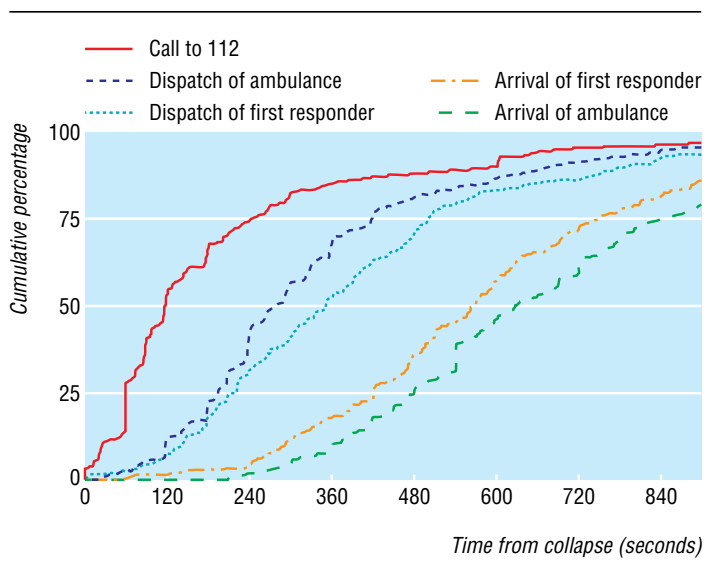

Fig 2 Cumulative time intervals from collapse to call, dispatch, and arrival of ambulance and first responders in the experimental group. Delays in placing the call by the witness and handling the call by the dispatcher contributed to over half of the total delay to arrival at the scene. Delay in dispatching the first responder eliminated much of the benefit of the shorter travel time of the first responder 


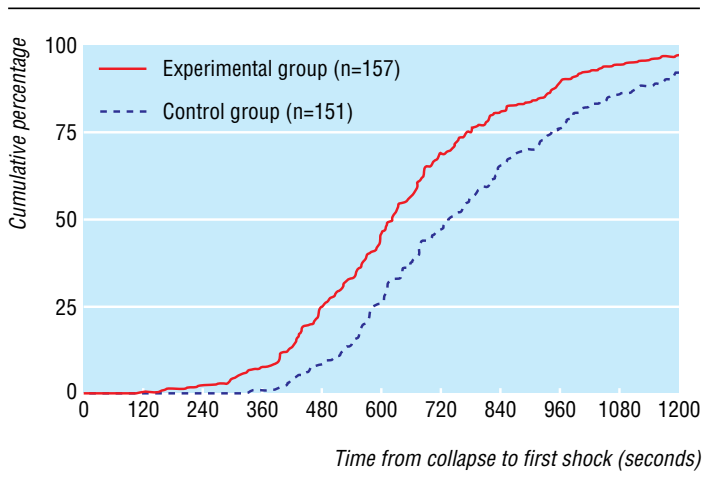

Fig 3 Cumulative time intervals from collapse to first shock for experimenta and control groups. In the experimental group the first shock was delivered by first responders or ambulance personnel, depending on who arrived first; this was the first responders in 72 cases and the ambulance in 85 cases. In the control group all shocks were delivered by the ambulance personnel. The difference between the two groups in the median time to first shock was 101 seconds

$85(54 \%)$ patients by the ambulance personnel. In the control group, all patients received their shocks from the ambulance personnel. The median time it took the first responder, after arrival at the scene, to deliver the first shock was 136 (interquartile range 83-183) seconds compared with 143 (94-215) seconds when the ambulance personnel delivered the first shock in the control group $(\mathrm{P}=0.07)$. The first shock was delivered in less than five minutes after call in $14(9 \%)$ patients in the experimental group and in $2(1 \%)$ patients in the control area $(\mathrm{P}<0.001)$. Overall, $7 / 16(44 \%)$ patients who received the first shock within five minutes after call survived to discharge.

\section{Use of the automated external defibrillator}

The first responders used the AED in 116 patients with a cardiac arrest. In $75(65 \%)$ patients the initial rhythm was shockable; the AED recognised this rhythm and advised a shock in 72 patients (sensitivity 96\%). In patients with an initial non-shockable rhythm, the AED never advised a shock (specificity 100\%). When first responders used the AED, median time to transfer to care by the emergency medical system was 2 minutes and 52 seconds.

\section{Discussion}

This prospective controlled study did not show a significant difference in survival to hospital discharge after introduction of automated external defibrillators used by first responders in a controlled programme. The secondary endpoints, return of spontaneous circulation and admission to hospital, were significantly improved, and a consistent relative benefit (odds ratio of 1.3-1.5) occurred in all three endpoints. The overall survival benefit was similar to the odds ratio of survival of 1.3 found for the total police AED programme in Miami-Dade County with a historical control, ${ }^{7}$ and much lower than the odds ratio of survival for witnessed arrests of 4.1 as reported in the Piacenza project with quasi-controls of cases in the same period in which the ambulance reached the patient first. ${ }^{8}$ The benefit was larger in the subgroup of patients with an initial shockable rhythm: the odds ratio was 2.1 in Miami-Dade County but was surprisingly reduced to 1.7 in Piacenza. Overall survival is the most relevant measure for assessing the benefit of a first responder AED programme, because the rhythm at first encounter is not independent of other factors in the programme such as time from collapse to first analysis of rhythm and cardiopulmonary resuscitation by a bystander. ${ }^{13}$
Several reasons could explain why our study showed only a modest survival benefit. The study was powered assuming a decrease of five minutes to first shock. The observed decrease in time to first defibrillation was only 101 seconds, which is partly explained by delays in communication between the emergency medical system and first responder dispatch centres. Survival models predict a 10-15\% reduction in survival for each minute's delay to defibrillation, in concordance with our observed survival difference..$^{14}$ In our study, the time to defibrillation was long, with a median time from collapse to shock of 668 seconds when first responders were equipped with an AED. With this small benefit in time to shock, a modest difference in survival to discharge between the experimental and control groups can be expected. The high percentage of cardiopulmonary resuscitation by bystanders and first responders before the arrival of the ambulance and the possible benefit of cardiopulmonary resuscitation before defibrillation could explain the high survival rate in the control group, thus reducing the power of the study. ${ }^{16}$

The proportion of deaths in hospital was similar in the experimental and control groups, suggesting that a larger study would have given a significant result in the primary endpoint. A controlled study powered for the observed survival difference would need 2400-4000 patients in each group. Such a study will be increasingly difficult to do as public access defibrillation becomes widely available throughout the Western world, ${ }^{17}{ }^{18}$ as the control group would then be contaminated by the off-protocol use of AEDs.

The median time from call to dispatch of the ambulance was two minutes in both groups. Use of a standardised interrogation protocol and computerised number and address recognition in dispatch centres could reduce this delay. ${ }^{19}$ Such delays are assumed to be short, but they may be common in dispatch systems and are rarely investigated..$^{20}{ }^{21}$

According to the 2000 guidelines for cardiopulmonary resuscitation and emergency cardiovascular care, delivery of the first shock within five minutes of receipt of a call to the emergency medical system is a high priority goal. ${ }^{4}$ In the experimental group, $9 \%$ of cases met this criterion versus $1 \%$ in the control group. In the experimental group, $20 \%$ would have had a shock delivered within five minutes of the call if first responders had been dispatched simultaneously with the ambulance.

\section{Limitations}

We achieved unbiased allocation of treatment by the systematic allocation of AEDs instead of randomisation by patient. The allocation of AEDs changed from control to experimental area every four months, and we presumed that the allocation of treatment was independent of the characteristics of the patients, thus closely meeting the objectives of true randomisation. With the statistical analysis method we used we tried to account for unmeasured factors related to region and period.

We included only patients with witnessed arrest, because only then could the time of collapse be estimated accurately. Non-witnessed arrest has a very low probability of survival, so the survival as presented overestimates the survival of out of hospital cardiac arrest in general. As the experimental treatment, use of the AED by first responders, could not be blinded, a potential bias existed in decision making and treatment of patients by the ambulance personnel. Not all arrests that occurred during the study period could be included in this study. On the basis of previous data, we estimate that about half of the arrests in the study region were not recognised as such by the dispatcher or the patient was not yet in arrest at the time of the call. 


\section{What is already known on this topic}

Reducing time to defibrillation improves survival after out of hospital cardiac arrest

Police officers and fire fighters can safely use an automated external defibrillator

\section{What this study adds}

This randomised trial could show only modest benefit of defibrillation by first responders

Delay in time to call, duration of call handling, and delay in dispatching severely reduce the potential benefit of dispatched first responders

An automated external defibrillator programme must focus on optimising the civic response and the dispatch process

\section{Conclusion}

Use of AEDs by first responders did not significantly increase survival to hospital discharge but did significantly improve return to spontaneous circulation and admission to hospital. Much of the potential benefit of dispatched first responders using AEDs was limited by time lost in decision making, incorrect decisions in the emergency medical dispatch centre, and delays in communication between dispatch centres. A dispatched first responder AED programme can achieve optimal benefit only when much attention is devoted to reducing time elements in the first link of the chain of survival, up to the arrival of first responders at the patient's side.

The steering committee is: R W Koster, Academic Medical Center, Amsterdam, Netherlands (chairman); L L Bossaert, University of Antwerp, Belgium; D Chamberlain, Hove, UK; A F Cohen, Center of Human Drug Research, Leiden, Netherlands; M Eisenberg, University of Washington, Seattle, WA, USA; R A Niskanen, Medtronic Physio-Control, Redmond, WA, USA (non-voting member). The data safety and monitoring committee is: J G P Tijssen, Academic Medical Center, Amsterdam (chairman); R de Vos, Academic Medical Center, Amsterdam; H J G M Crijns, University of Maastricht, Netherlands; J Herlitz, Göteborg University, Sweden.We thank the participants in the dispatch centres for their efforts and cooperation, the police officers of the police district Kennemerland and ZaanstreekWaterland, the fire fighters of the fire brigades of Amsterdam and Amstelveen, and the paramedics of the participating emergency medical system for their dedication. We also thank the medical students and C M Visscher-van der Burg for their work in data collection, I Vrenken-Witte for technical support, and J B Reitsma for statistical support.

Contributors: APvA took part in collecting, analysing, and interpreting the data and drafted the manuscript. RHV and RdV took part in the conception, design, and planning of the study, in the data collection, and in revising the manuscript.JGPT took part in the design and conception of the study and has revised the manuscript critically for important intellectual content and chaired the data safety and monitoring committee. RWK conceived the study, supervised the data collection, assisted in the interpretation of the data, took part in drafting the manuscript, revised it critically for important intellectual content, and chaired the steering committee. RWK as guarantor accepts full responsibility for the conduct of the study, had access to the data, and controlled the decision to publish. Funding: Grant from the Netherlands Heart Foundation (grant 98.179) and an unrestricted grant of Medtronic Physio-Control, Redmond, WA, USA. Neither source of funding had a role in study design; in the collection, analysis, and interpretation of data; in the writing of the report; or in the decision to submit the paper for publication.
Competing interests: RWK received material and financial support from Medtronic Physio-Control in organising the study.

Ethical approval: Medical ethics committees from participating hospitals and the emergency medical system approved the study and considered the study exempt from informed consent before treatment. Medical authorisation was obtained from patients or family members after resuscitation, and all consented to provide access to their medical records.

1 Cummins RO, Eisenberg M, Bergner L, Murray JA. Sensitivity, accuracy, and safety of an automatic external defibrillator. Lancet 1984;2:318-20.

2 Cummins RO, Eisenberg MS, Stults KR. Automatic external defibrillators: clinical issues for cardiology. Circulation 1986;73:381-5.

3 Jaffe A, Landau WM, Wetzel RD. Automated external defibrillators. $N$ Engl J Med 2001;344:771-3.

European Resuscitation Council. Part 4: the automated external defibrillator: key link in the chain of survival. Resuscitation 2000;46:73-91.

5 White RD, Asplin BR, Bugliosi HF, Hankins DG. High discharge survival rate after outof-hospital ventricular fibrillation with rapid defibrillation by police and paramedics. of-hospital ventricular fibrillation

6 Mosesso VN Jr, Davis EA, Auble TE, Paris PM, Yealy DM. Use of automated external defibrillators by police officers for treatment of out-of-hospital cardiac arrest. Ann Emerg Med 1998;32:200-7.

7 Myerburg RJ, Fenster J, Velez M, Rosenberg D, Lai S, Kurlansky P, et al. Impact of community-wide police car deployment of automated external defibrillators on survival from out-of-hospital cardiac arrest. Circulation 2002;106:1058-64.

8 Capucci A, Aschieri D, Piepoli MF, Bardy GH, Iconomu E, Arvedi M. Tripling survival from sudden cardiac arrest via early defibrillation without traditional education in cardiopulmonary resuscitation. Circulation 2002;106:1065-70.

9 Waalewijn RA, de Vos R, Koster RW. Out-of-hospital cardiac arrests in Amsterdam and its surrounding areas: results from the Amsterdam resuscitation study (ARREST) in its surrounding areas: results from the Am
'Utstein' style. Resuscitation 1998:38:157-67.

10 Diggle PJ, Liang KY, Zeger SL. Analysis of longitudinal data. Oxford: Oxford University Press, 1994

11 Zeger SL, Liang KY. Longitudinal data analysis for discrete and continuous outcomes. Biometrics 1986;42:121-30.

12 Handley J, Negassa A, Edwardes MD, Forrester JE. Statistical analysis of correlated data using generalized estimation equations: an orientation. Am J Epidemiol 2003;157:36475 .

13 Waalewijn RA, Nijpels MA, Tijssen JG, Koster RW. Prevention of deterioration of ventricular fibrillation by basic life support during out-of-hospital cardiac arrest. Resuscitation 2002;54:31-6.

14 Valenzuela TD, Roe DJ, Cretin S, Spaite DW, Larsen MP. Estimating effectiveness of cardiac arrest interventions: a logistic regression survival model. Circulation cardiac arrest in
$1997 ; 96: 3308-13$.

15 Waalewijn RA, de Vos R, Tijssen JG, Koster RW. Survival models for out-of-hospital cardiopulmonary resuscitation from the perspectives of the bystander, the first responder, and the paramedic. Resuscitation 2001;51:113-22.

16 Cobb LA, Fahrenbruch CE, Walsh TR, Copass MK, Olsufka M, Breskin M, et al. Influence of cardiopulmonary resuscitation prior to defibrillation in patients with out-of-hospital ventricular fibrillation. JAMA 1999;281:1182-8.

17 Ornato JP, Hankins DG. Public-access defibrillation. Prehosp Emerg Care 1999;3:298302.

18 Davies C, Colquhoun M, Graham S, Evans T, Chamberlain D, Defibrillator Advisory Committee. Defibrillators in public places: the introduction of a national scheme for public access defibrillation in England. Resuscitation 2002;52:13-21.

19 Eisenberg MS, Carter W, Hallstrom AP, Cummins R, Litwin P, Hearne T. Identification of cardiac arrest by emergency dispatchers. Am J Emerg Med 1986;4:299-301.

20 Ross P, Nolan J, Hill E, Dawson J, Whimster F, Skinner D. The use of AEDs by police officers in the City of London: automated external defibrillators. Resuscitation 2001;50:141-6.

21 Groh WJ, Newman MM, Beal PE, Fineberg NS, Zipes DP. Limited response to cardiac arrest by police equipped with automated external defibrillators: lack of survival benefit in suburban and rural Indiana-the police as responder automated defibrillation evaluation (PARADE). Acad Emerg Med 2001;8:324-30.

(Accepted 26 September 2003)

bmj.com 2003;327:1312

Department of Cardiology, Academic Medical Center, Meibergdreef 9, 1105 AZ Amsterdam, Netherlands

Anouk P van Alem junior scientist

Jan G P Tijssen clinical epidemiologist

Rudolph W Koster lecturer in cardiology

Department of Clinical Epidemiology and Biostatistics, Academic Medical Center, Amsterdam

Rien de Vos clinical epidemiologist

Municipal Health Service, Amsterdam

Rob H Vrenken medical director, municipal health service

Correspondence to: A P van Alem

a.p.vanalem@amc.uva.nl 